\title{
Effect of Cisplatin Arterial Infusion (CAI) on Primary Nonmetastatic Pelvic Osteosarcoma: A Preliminary Study
}

This article was published in the following Dove Press journal: Cancer Management and Research

\section{Xuantao Hu iD \\ Xia Chen \\ Tao Li iD \\ Zicheng Liu \\ Xiaoning Guo \\ Zhengxiao Ouyang (D) \\ Department of Orthopedics, The Second Xiangya Hospital of Central South University, Changsha, Hunan, 4I00II, People's Republic of China}

Correspondence: Xiaoning Guo;

Zhengxiao Ouyang

Department of Orthopedics, The Second Xiangya Hospital of Central South University, 139 Renmin Road, Changsha, 4I00II, Hunan, People's Republic of China

Tel +86-073 I-85295I 27

Email guoxiaoning@csu.edu.cn; ouyangzhengxiao@csu.edu.cn
Purpose: The critical role of arterial infusion chemotherapy in the multimodal treatment of extremity bone cancer has been investigated extensively, but few studies have focused on pelvic osteosarcoma. Therefore, we attempted to evaluate the clinical significance of arterial infusion chemotherapy in the treatment of pelvic osteosarcoma.

Patients and Methods: We combined a cisplatin arterial infusion regimen with multidrug systematic chemotherapy as a neoadjuvant protocol for the treatment of pelvic osteosarcoma. The course number and dosage of cisplatin arterial infusion were adjusted to achieve a maximal tumor response evaluated by contrast-enhanced MRI per RECIST 1.1. Good responders received the same systematic combination for postoperative chemotherapy, and poor responders received second-line therapy. Twelve patients with nonmetastatic high-grade pelvic osteosarcoma were included. Survival, chemotherapy response and adverse events data were analyzed.

Results: The mean follow-up period was 56.1 months. Four patients died of refractory tumor progression, and 1 patient with local recurrence had no evidence of disease for 27 months after receiving secondary amputation and resection. Kaplan-Meier survival analysis demonstrated a $57.8 \%$ overall survival and $52.5 \%$ event-free survival rate at 5 years. Eight of 12 patients had a $>90 \%$ tumor necrosis rate according to histopathologic examinations. The rates of local adverse events were lower than those reported for extremity osteosarcoma.

Conclusion: Our study initially indicated that the cisplatin arterial infusion regimen was a potential therapy with good tolerance in the treatment of pelvic osteosarcoma.

Keywords: osteosarcoma, pelvis, cisplatin, arterial infusion, chemotherapy toxicity

\section{Introduction}

Osteosarcoma is the most common solid malignant tumor of bone with a highly aggressive behavior. ${ }^{1}$ The most susceptible age of osteosarcoma is bimodal, which is characterized by one peak at 10-to-20 years and another at over 60 years., Although this disease endangers the health of both adolescent and aged populations, limited progress has been made to improve survival and functional prognosis, especially in patients with pelvic osteosarcoma. ${ }^{4-6}$ Osteosarcoma patients treated with mere immediate surgical resection register a recurrence rate up to $60-90 \%{ }^{7}$ (Since 1980s, traditional adjuvant intravenous (IV) systematic chemotherapy produces an improved five-year event-free survival (EFS) rate of $61 \% .^{8}$ In the early 2000 s, the propagation of a multidrug neoadjuvant chemotherapy regimen elevated this rate to $60 \%$ and above. ${ }^{9,10}$ ) Henceforth, numerous explorations have been 
conducted to optimize the category, dosage, combination and duration of chemotherapy drug administration. The most recent studies of EURAMOS revealed a 5-year EFS of $65-70 \%$ for localized osteosarcoma treated by multidrug neoadjuvant chemotherapy. However, there are few studies focusing on arterial infusion as an improved method and its clinical outcome in the treatment of pelvic osteosarcoma.

Osteosarcoma in pelvic bone is more notorious than that in extremities. Patients with pelvic osteosarcoma have an approximately $30 \%$ lower survival rate than those with extremity lesions, which may be associated with a poor chemotherapy response and an inability to achieve adequate surgical resection. ${ }^{11}$ Therefore, more challenges are faced in the management of pelvic osteosarcoma.

Platinum complexes, such as cis-diamine-dichloro platinum (cisplatin, CDDP), have been proven to be cell cycle-nonspecific agents and to have multiple broadspectrum antitumor effects. ${ }^{12}$ Several clinical trials reported that employing an arterial infusion technique to deliver CDDP, in combination with an IV systematic chemotherapy regimen or not, can elevate the local drug concentration to fivefold that of IV administration and can produce better survival in the treatment of extremity osteosarcoma. ${ }^{13-15}$ Therefore, the arterial infusion of CDDP might have potential in the treatment of pelvic osteosarcoma.

The histopathologic response of surgically removed tumors has been shown to be one of most critical indicators of chemotherapeutic effectiveness and survival prognosis. ${ }^{16,17}$ However, preoperative examination is more valuable to predict tumor response and allow individualized neoadjuvant chemotherapy. Response evaluation criteria in solid tumors version 1.1 (RECIST 1.1), which was commonly adopted for the tumor-response evaluation of a variety of cancers, affirmed the value of sectional radiological imaging measurement. ${ }^{18-20}$ Therefore, preoperative contrast-enhanced MRI (CE-MRI) evaluation based on RECIST 1.1 is expected to guide the adjustment of treatment for optimal surgical timing in pelvic osteosarcoma.

Given all these findings, we combined CDDP arterial infusion (CAI) with individualized multidrug systematic chemotherapy (MSC) and surgery (S) to treat nonmetastatic pelvic osteosarcoma and attempted to investigate (1) the chemotherapy response and survival yielded from this protocol, (2) the individualized adjustment of the neoadjuvant MSC regimen necessary to achieve the maximal tumor response according to RECIST-1.1-based CE-MRI reassessment, and (3) the potential adverse effects caused by the arterial infusion technique and the multidrug systematic chemotherapy regimen.

\section{Patients and Methods Study Design and Consent}

This is a retrospective cohort study (Level III evidence) in which subjects received a protocol comprising multidrug systematic chemotherapy, cisplatin arterial infusion and resection surgery $(\mathrm{CAI}+\mathrm{MSC}+\mathrm{S})$. The study design conformed with the Declaration of Helsinki, and the treatment protocol was approved by the hospital institutional review board. All patients provided written informed consent for receiving this treatment protocol. All data used in the present study were obtained from the electronic medical records of our hospital and treated with strict confidentiality; therefore, the requirement of obtaining specific informed consent for this retrospective study was waived.

\section{Patients}

Inclusion criteria for subjects included (1) histopathologically defined primary osteosarcoma of pelvic bone; (2) no metastatic foci; (3) no past history of cancer treatment; and (4) normal cardiac, hepatic and renal function. All patients were confirmed to have high-grade osteosarcoma morbidity via biopsy. Pretreatment baseline safety indexes were determined with routine blood tests, hepatic and renal function tests, and Doppler echocardiography. A peripherally inserted central catheter was placed in each patient for the IV administration of neoadjuvant chemotherapy agents.

From January 2010 to June 2015, 21 patients were consecutively diagnosed with osteosarcoma of the pelvic bones in our work unit. A total of 4 patients were excluded for the following reasons: 2 patients had evidence of distant metastasis, and 2 tumors involved the femur or lumbar vertebra. Among the eligible people, 2 refused to take CAI + MSC + S treatment: 1 had hemophilia with severely impaired coagulation function; 1 intended to transfer to another hospital after being definitively diagnosed via biopsy. Subsequently, 15 patients took the CAI + MSC + $\mathrm{S}$ treatment, but 1 of them stopped midway (because of intolerance to vomiting as the adverse effect in the first two rounds of IV multidrug therapy) and turned to other treatment. Two patients were lost to follow-up after surgical resection. Eventually, 12 patients finished the treatment 
protocol and yielded analyzable data for survival, chemotherapy response and toxicity assessment.

The data of these 12 patients are listed in Table 1. The study population consisted of $7(58.3 \%)$ males and 5 (41.7\%) females with an average age of 28.8 years (11 to 69 years). The morbid sites included the pubis $(\mathrm{n}=2)$, ilium $(n=4)$, ischium $(n=1)$, sacrum $(n=1)$ and unclear sites $(n=4)$.

\section{Description of the Study Treatments and Outcome Measures}

The adopted CAI $+\mathrm{MSC}+\mathrm{S}$ treatment protocol is depicted in Figure 1. In general, MSCs usually consist of high-dose methotrexate (MTX), vincristine (VCR), adriamycin (ADM), CDDP and ifosfamide (IFO). Specifically, the routine CAI + MSC regimen was initiated with 24hour continuous IV dripping of high-dose MTX at $10 \mathrm{~g} / \mathrm{m}^{2}$ followed by $2 \mathrm{mg}$ IV VCR the next day for the first group at Week 0 ; in Week 2, $120 \mathrm{mg} / \mathrm{m}^{2}$ CDDP AI over 3 to 6 hours and following $30 \mathrm{mg} / \mathrm{m}^{2} / \mathrm{d}$ IV ADM for 2 days were given to the second group; these 2 groups were repeated in Weeks 3 and 4; then, the resection surgery was performed 2 weeks after the neoadjuvant chemotherapy was finished. Notably, $160 \mathrm{mg} / \mathrm{m}^{2}$ was determined as the enhanced dosage of CDDP for tumors with the longest diameter over $8 \mathrm{~cm}$ in CE-MRI slices. In addition, IV IFO at $2 \mathrm{~g} /$ $\mathrm{m}^{2} / \mathrm{d}$ for 5 days was given in Week 5 for tumors over $8 \mathrm{~cm}$ after the 4-week routine regimen was completed. Central venous catheterization for MSC administration was performed under inhalation anesthesia if necessary. Before each CAI regimen, percutaneous punctuation of the femoral artery was performed using the Seldinger technique, and the catheter was inserted from the femoral artery of the contralateral side to the beginning of the artery trunk supplying the tumor with the assistance of CT-guided positioning. ${ }^{21}$ Then, CT arterial angiography was conducted. Each course of MSC and CAI was accompanied by IV hydration, diuretics, rescue and protecting drugs. The CDDP was perfused via the catheter with chemotherapy pumps (LimLess Infusion Pump; PFM Medical Inc, Cologne, Germany) in a pulsed manner (Bolus dose administrator; PFM Medical Inc, Cologne, Germany). The patients were asked to have strict bed rest under multiparameter monitoring. Routine blood tests for electrolytes and hepatic and renal function were performed on the first day after drug administration and every 3 days thereafter.
To evaluate the therapeutic response, primary plain $\mathrm{X}$-ray (Figure 2A) and physical examination parameters of the tumor, including pre- and postchemotherapy size, tenderness, pain and inflammation, were collected. More importantly, CE-MRI was performed to monitor the tumor chemotherapy response before and after routine neoadjuvant CAI + MSC administration. (Figure 2B-E) According to the RECIST 1.1 guidelines, tumor size was calculated in gadolinium-enhancing T1-weighted fat-saturated coronal images using the following criteria: complete response (CR), complete elimination of target lesion; partial response (PR), $\geq 30 \%$ decrease in the diameter of target lesion compared to baseline); progressive disease (PD), $\geq 20 \%$ increase in the diameter of target lesion compared to baseline or new metastatic lesion); and stable disease (SD) or observed changes in the diameter of the target lesion between $30 \%$ decrease and $20 \%$ increase compared to baseline. Each tumor lesion was assessed by orthopedic oncologists and interventional radiologists who were blinded to the treatment allocation and clinical outcome data. For patients who achieved PD after the routine neoadjuvant regimen was finished, surgery was performed immediately. For patients who demonstrated CR or PR, surgery was performed 2 weeks after the neoadjuvant regimen as usual. For patients who presented SD, the same IFO course as that mentioned in the adjustment for large tumors was added to maximize the tumor response of the neoadjuvant regimen. Then, a multidisciplinary consultation was conducted to identify the achievement of maximal tumor response. If any uncertainty of maximal response achievement was left, one more course of IFO could be given, and patients received resection surgery no later than 9 weeks after the first diagnosis.

A chest CT scan, a full-body bone scan and a pelvic CE-MRI scan (Figure 2D and E) were repeated before surgery. The tumor response per RECIST 1.1 measured in the last CE-MRI examination was compared with the baseline level. Surgical excision extension was determined by the involved scope of the tumor and the tumor response category according to the CE-MRI assessment per RECIST 1.1. The pelvic reconstruction project depended on the morbidity site, excision extension, bone mineral density and functional requirements.

In case of uncontrollable hemorrhage during the resection surgery, a temporary balloon blocking technique for the abdominal aorta was performed under the guidance of CTA 4 hours before resection surgery. A balloon compatible in size to the abdominal aortic diameter was placed 


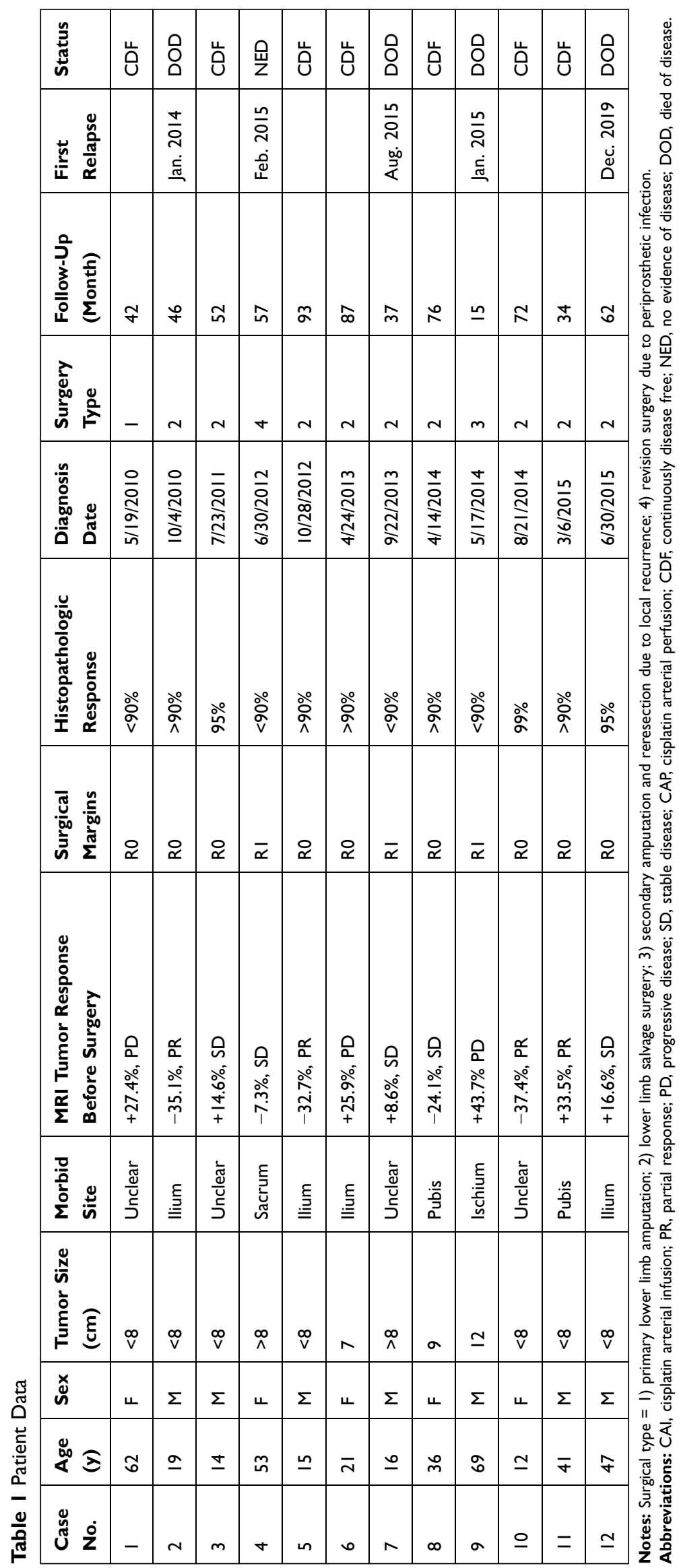




\section{The CAI + MSC + S treatment protocol for pelvic osteosarcoma patients}

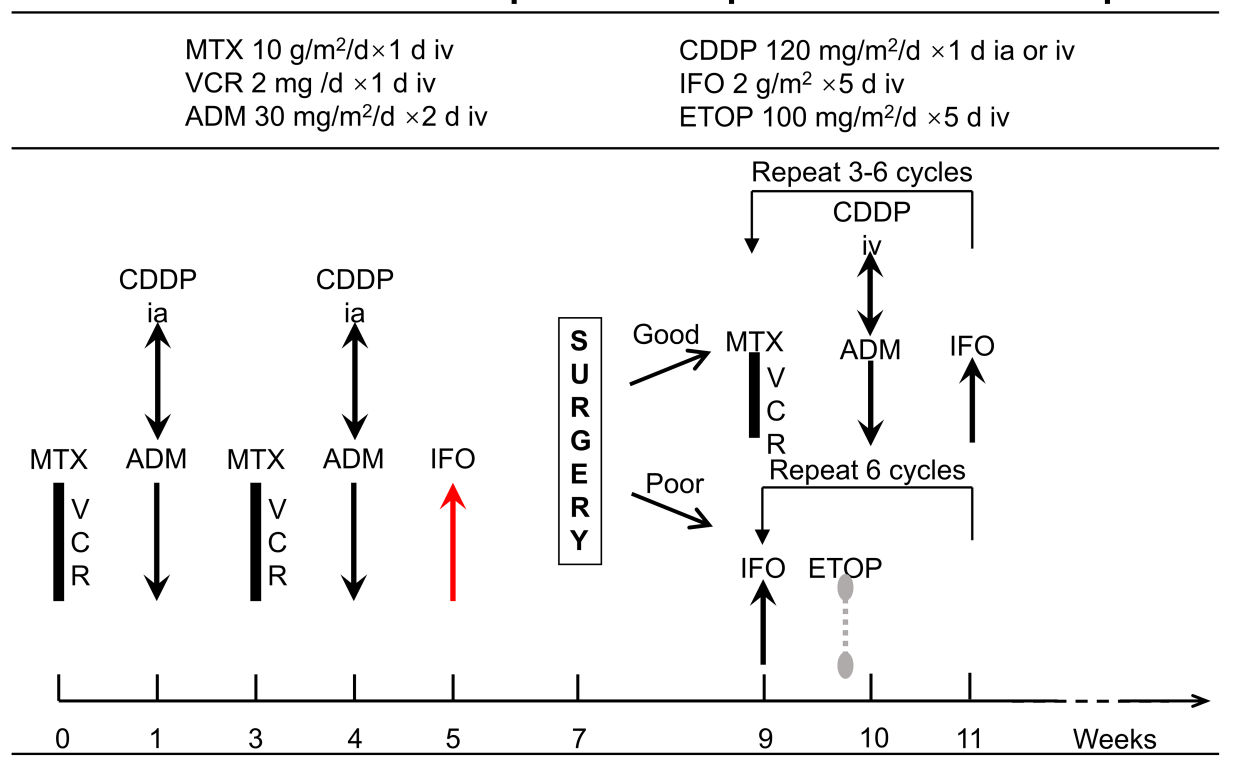

Figure I The individualized pelvic osteosarcoma treatment protocol combined cisplatin arterial infusion, multidrug systematic chemotherapy and resection surgery (CAI + $\mathrm{MSC}+\mathrm{S})$.

intraarterially at the level of 2 to $3 \mathrm{~cm}$ above the bifurcation of the common iliac arteries. The injection of saline with $0.1 \%$ heparin inflated the balloon, consequently blocking blood perfusion of the surgical site. Angiography was performed to confirm the satisfactory blockage of the iliac arteries and unobstruction of the renal arteries. Then, the balloon was deflated until the surgery began. Each deflation was performed with an interval of $60 \mathrm{~min}$ during the resection surgery before satisfactory hemostasis was achieved.

Histopathologic examination was conducted using the method reported by Huvos et al to quantify the necrosis ratio of the tumor after removal. ${ }^{22}$ Patients with a $\geq 90 \%$ tumor necrosis rate (TNR) (Grade III and IV) were classified as good responders. The other (Grade I and II) were deemed poor responders.

The postoperative IV chemotherapy cycle was repeated every 3 weeks. The recovery of hematopoietic function in patients was required before each course, which was defined as a white blood cell count over $1 \times 10^{4} / \mathrm{mm}^{3}$ and a platelet count over $5 \times 10^{6} / \mathrm{mm}^{3}$ for 3 successive days without hematopoietic stimulants. For good responders, the duration of postoperative MSC was usually 3 courses, which could be extended to the maximum of 6 courses accompanied by the existence of high-risk factors, including SD or PD in the CE-MRI per RECIST 1.1 before surgery or histology-proven tumor involvement in the surgical margin (R1 resection). For poor responders, IFO at $2 \mathrm{~g} / \mathrm{m}^{2} / \mathrm{d}$ for 5 days and etoposide (ETOP) at $100 \mathrm{mg}$ / $\mathrm{m}^{2} / \mathrm{d}$ for 5 days were given sequentially as one cycle repeated every 3 weeks for 6 cycles. $^{23}$

After the planned treatment was finished, the discontinuation of chemotherapy was allowed by the orthopedic oncologist if X-ray (Figure 2F) and CE-MRI of the primary morbidity site along with chest X-ray every 2 months, chest CT scanning every 6 months in the first year off the treatment prove no local recurrence or lung metastasis. Routine blood tests and hepatic and renal function tests were deployed every week until the recovery of hematopoietic function.

\section{Statistical Analysis}

We used the Kaplan-Meier method to analyze the overall survival (OS) and EFS, which were calculated from the day the preoperative MSC + CAI regimen began. The events were defined as local recurrence, distant metastasis or death from the disease. Additionally, the OS and EFS in groups with different histopathologic chemotherapy responses or tumor sizes were calculated. All statistical analyses were conducted with SPSS software packages (SPSS Inc., Chicago, IL, USA).

\section{Results}

\section{Chemotherapy Response}

Significant improvement in physical examination parameters was observed in 10 of 12 patients after the 

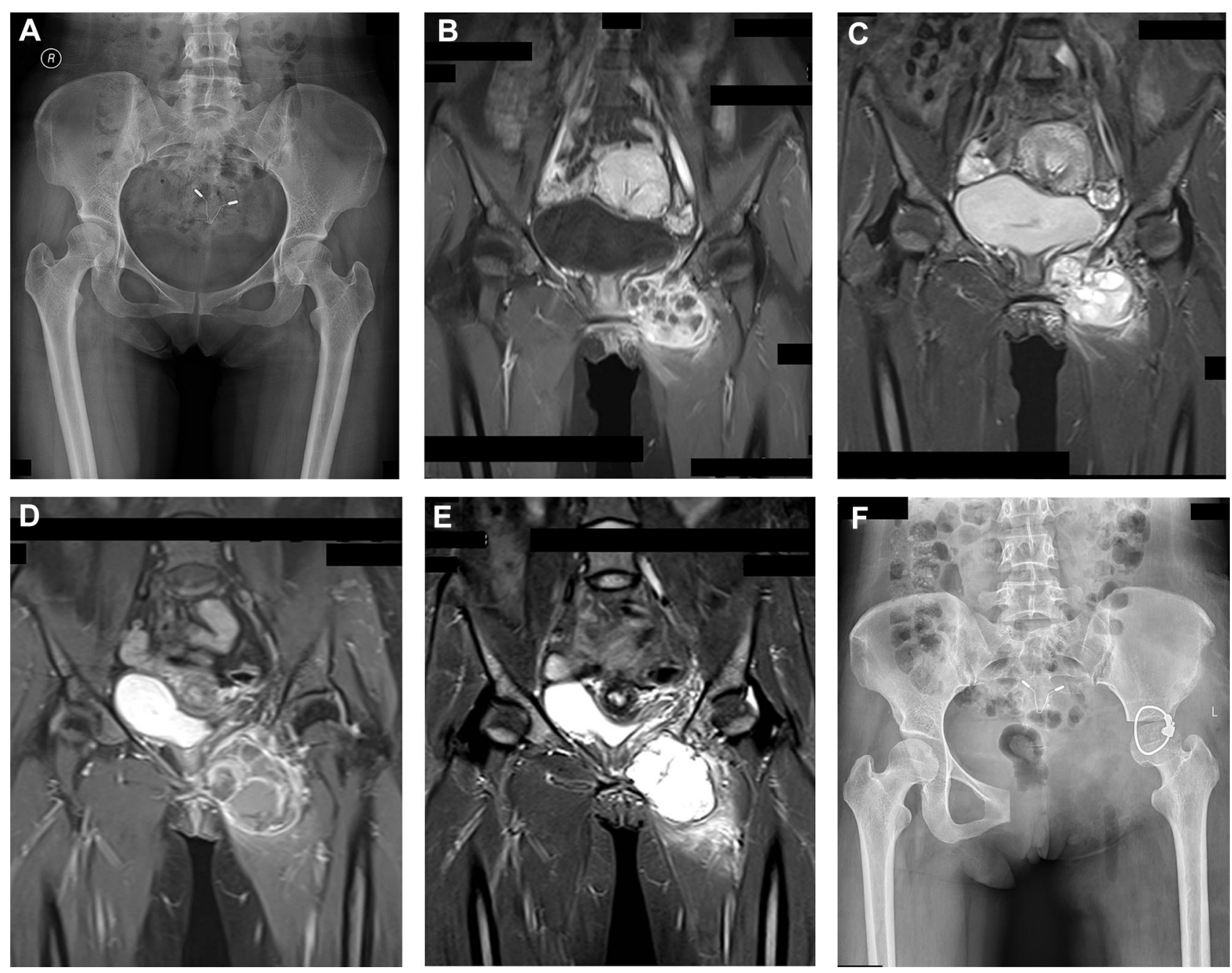

Figure 2 (A) Pelvis X-ray of a 36-year-old female with an osteosarcoma in the pubis. (B) and (C) Fat-saturated TI- and T2-weighted coronal slices of contrast-enhanced MRI showing the maximum diameter of the tumor at the first diagnosis, which showed heterogeneous enhancement, thick septa and obscure boundaries of the tumor. (D) and (E) MRI reevaluation after completion of the neoadjuvant CAI + MSC regimen, showing thin septa, clear boundaries and stable disease according to RECIST I.I with a decrease in the tumor diameter. (F) Pelvis plain X-ray of this patient. (The identifiable captions in these images are occluded.).

neoadjuvant regimen, including alleviation of pain. In terms of tumor response measurement per RECIST 1.1, 4 patients had PR and 5 had SD after routine neoadjuvant CAI + MSC administration. Three patients had to undergo resection surgery ahead of schedule because of $\mathrm{PD}$, as shown by CE-MRI after neoadjuvant CAI + MSC administration. No patient demonstrated CR.

According to the histopathologic assessment, 66.7\% (8 of 12) of patients demonstrated TNR over $90 \%$. One of the 3 patients with PD according to the CE-MRI assessment turned out to be good responders with Grade III histopathologic results.

\section{Surgery}

Three patients underwent local radical excision and lower limb amputation because of neurovascular bundle involvement. The remaining 9 patients received limb salvage surgery followed by pelvic reconstruction using a modular prosthesis (Lidakang Inc., Beijing, China), tumor bone devitalization and replantation, or just bone fusion. As a result, 1 patient who underwent primary limb salvage surgery developed local recurrence and underwent secondary amputation and local reresection. Two patients had surgical site infections but were cured 2 months after surgery. One patient who developed a periprosthetic infection underwent a secondary amputation.

\section{Survival}

A total of $58.3 \%$ of patients ( 7 of 12 ) remained continuously disease free in an average follow-up period of 65.1 months (range, 34-93 months). One patient who developed local recurrence was proven alive with no evidence of 


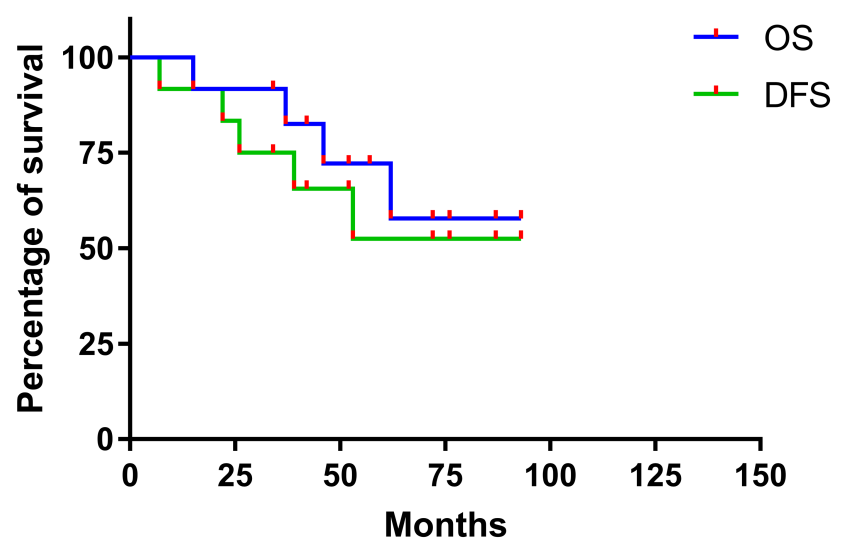

Figure 3 The Kaplan-Meier survival curve for overall survival (OS) and event-free survival (EFS) rates of 12 pelvic osteosarcoma patients treated with the CAI + MSC + S protocol.

disease for 31 months after receiving a secondary amputation and resection. Four patients (3 lung metastases and 1 local recurrence) died of refractory disease progression. Kaplan-Meier survival analysis suggested an OS rate of $57.8 \%$ and an EFS of $52.5 \%$ at 5 years (Figure 3). For patients with a good chemotherapy response, the 5-year OS and EFS rates were $68.6 \%$, both of which showed significant differences ( $\mathrm{P}=0.04$ for OS and 0.01 for EFS) compared with the corresponding measures for poor responders (Table 2). The comparison of patients with tumor sizes

Table 2 Overall Survival (OS) and Event-Free Survival (EFS) Rate

\begin{tabular}{|l|l|l|}
\hline Variables & $\mathbf{I} \mathbf{y}$ & $\mathbf{5}$ y \\
\hline All patients - OS & $91.7 \%$ & $57.8 \%$ \\
\hline All patients - EFS & $91.7 \%$ & $52.5 \%$ \\
\hline Good responders - OS & $100.0 \%$ & $68.6 \%$ \\
\hline Good responders - EFS & $100.0 \%$ & $68.6 \%$ \\
\hline Poor responders - OS & $75.0 \%$ & $50.0 \%$ \\
\hline Poor responders - EFS & $75.0 \%$ & $25.0 \%$ \\
\hline Patients with tumor size $\geq 8 \mathrm{~cm}-$ OS & $75.0 \%$ & $50.0 \%$ \\
\hline Patients with tumor size $\geq 8 \mathrm{~cm}-$ EFS & $75.0 \%$ & $25.0 \%$ \\
\hline Patients with tumor size<8 cm - OS & $100.0 \%$ & $62.5 \%$ \\
\hline Patients with tumor size<8 cm - EFS & $100.0 \%$ & $64.3 \%$ \\
\hline R0 resection - OS & $85.7 \%$ & $70.0 \%$ \\
\hline R0 resection - EFS & $88.9 \%$ & $71.1 \%$ \\
\hline RI resection - OS & $66.7 \%$ & $33.3 \%$ \\
\hline RI resection - EFS & $66.7 \%$ & $33.3 \%$ \\
\hline Note Confide Interv $95 \%$ & & \\
\hline
\end{tabular}

Note: Confidence Interval $=95 \%$ greater $(\mathrm{n}=4)$ or less $(\mathrm{n}=8)$ than $8 \mathrm{~cm}$ did not yield statistical significance ( $\mathrm{P}=0.32$ and 0.13 , respectively). As shown in the pathological report, $75 \%$ (9 of 12) patients received R0 resection and 25\% (3 of 12) had R1 resection. There was no significant difference in the OS but in the EFS between patient group with $\mathrm{R} 0$ or $\mathrm{R} 1$ surgical resection $(\mathrm{P}=$ 0.15 and 0.05 , respectively). The confidence interval of all OS and EFS are $95 \%$.

\section{Chemotherapy Toxicity}

We recorded the adverse effects referred to in the Common Terminology Criteria for Adverse Events (CTCAE) version 5.0. ${ }^{24}$ The statistics of CAI-related adverse events are summarized in Table 3. Three of 12 patients had local adverse events, including grade 1 to 2 skin pelvic soft tissue necrosis in muscle and hyperpigmentation, which exhibited no effect on the surgical method choice or wound healing. Nausea and vomiting were the most common systematic adverse events, with $41.7 \%$ of patients developing grade 3 events. A 36-year-old female received a reduced dosage of MTX $\left(7 \mathrm{~g} / \mathrm{m}^{2}\right)$ due to intolerable vomiting after the first MTX course. Four of 12 patients developed grade 1 infusion-related reactions.

During the postoperative MSC period, one 12-year-old child complained of grade 1 palpitations and chest pain. Therefore, we replaced ADM with ETOP, and the complaint disappeared with no evidence reported in Doppler echocardiography. Three patients out of 12 developed grade 1 to 2 increases in alanine/aspartate aminotransferase levels.

Table 3 Statistics of CAI-Related Adverse Events

\begin{tabular}{|c|c|c|}
\hline Adverse Events & $\begin{array}{l}\text { No. of } \\
\text { Courses }\end{array}$ & $\begin{array}{l}\text { Percentage of } \\
\text { Patients with } \\
\text { Grade 3-4 } \\
\text { Adverse Events }\end{array}$ \\
\hline Skin hyperpigmentation & $12.5 \%(3 / 24)$ & $0 \%(0 / 12)$ \\
\hline $\begin{array}{l}\text { Pelvic soft tissue necrosis } \\
\text { (muscle) }\end{array}$ & $4.2 \%(1 / 24)$ & $0 \%(0 / I 2)$ \\
\hline Pelvic pain & $16.7 \%(4 / 24)$ & $8.3 \%(1 / / 2)$ \\
\hline Nausea and vomiting & $75.0 \%(18 / 24)$ & $41.7 \%(5 / 12)$ \\
\hline Anemia & $8.3 \%(2 / 24)$ & $8.3 \%(1 / / 2)$ \\
\hline Increase in creatinine level & $8.3 \%(2 / 24)$ & $0 \%(0 / 12)$ \\
\hline $\begin{array}{l}\text { Increase in aminotransferase } \\
\text { level }\end{array}$ & $4.2 \%(1 / 24)$ & $0 \%(0 / 12)$ \\
\hline Infusion related reaction & $29.2 \%(7 / 24)$ & $0 \%(0 / 12)$ \\
\hline
\end{tabular}


Three children developed oral mucositis at grade 2 to 3,2 of whom were under IV nutrition, and one used a mouth rinse with metronidazole to treat thrush. All patients eventually resumed normal oral food intake.

Blood system disorders were commonly noted in patients after the second cycle of postoperative MSC administration. Three patients developed febrile neutropenia and were treated by injection of recombinant human granulocyte stimulating factor.

\section{Discussion}

Conventional IV adjuvant chemotherapy and neoadjuvant chemotherapy have been applied extensively in multimodal osteosarcoma treatment for over 45 years. ${ }^{25}$ Though changes in drug combination, dosage, and administration duration were made to optimize the regimen, no significant improvement was achieved based on the approximately $50 \%$ to $70 \%$ 5-year OS and EFS rates in the past decade. ${ }^{26-28}$ As a modified approach to augment the potential of chemotherapy drugs, the AI technique combined with MSCs has been studied for years. ${ }^{13,29,30}$ In the present study, we attempted to investigate the therapeutic effect of the CAI + MSC + $\mathrm{S}$ protocol in osteosarcoma treatment. The 5-year OS and EFS rates were $57.8 \%$ and $52.5 \%$, respectively.

The CAI regimen and its outcome measurements were first documented by Jaffe et al in $1983 .{ }^{31}$ Then, a series of studies concerning CAI-facilitated multidrug chemotherapy followed. ${ }^{13,30,32-37}$ Information on all 9 studies, including the present study, is listed in Table 4.

Among these 9 studies, 8 used CAI and systematic chemotherapy sequentially before surgery, and 5 studies scheduled a 2-course preoperative regimen. Six of 9 studies suggested a higher chemotherapy response rate and/or survival rate in the CAI group compared with the IV CDDP group; 2 of these investigations were 2-arm randomized cohort studies and reported a statistically significant improvement in the good-response ratio $(21 \%$ and $31 \%$, respectively). In the third randomized cohort study reported by Rha et al, the improvement was not statistically significant, probably because the limited sample size

Table 4 Synopsis of IA CDDP Information Reported in the Literature

\begin{tabular}{|c|c|c|c|c|c|c|c|c|c|}
\hline Authors & Type of Study & $\begin{array}{l}\text { No. of } \\
\text { Patients }\end{array}$ & $\begin{array}{l}\text { Ages } \\
\text { (y) }\end{array}$ & Sites & Metastases & $\begin{array}{l}\text { Preoperative } \\
\text { CDDP } \\
\text { Courses No. }\end{array}$ & $\begin{array}{l}>90 \% \\
\text { TN }\end{array}$ & os & EFS \\
\hline $\begin{array}{l}\text { Winkler et al } \\
\text { and Fuchs } \\
\text { et } \mathrm{al}^{30,34}\end{array}$ & $\begin{array}{l}\text { Nonrandomized, } \\
2 \text { arms }\end{array}$ & 50 & $<40$ & All & Included & 2 & $68 \%$ & $67 \%$ at $10 y$ & $63 \%$ at $10 y$ \\
\hline Ferrari et $\mathrm{al}^{33}$ & Single arm & 164 & $<40$ & Extremity & Excluded & 2 & NA & $72 \%$ at $8 y$ & $63 \%$ at $8 y$ \\
\hline Ferrari et $\mathrm{al}^{33}$ & $\begin{array}{l}\text { Randomized, } 2 \\
\text { arms }\end{array}$ & 59 & $<40$ & Extremity & Excluded & 2 & $64 \%$ & $61 \%$ at $8 y$ & $54 \%$ at $8 y$ \\
\hline Rha et $\mathrm{al}^{35}$ & Single arm & 37 & $8 \sim 41$ & Extremity & $?$ & 3 & $75 \%$ & $78 \%$ at $3 y$ & $55 \%$ at $3 y$ \\
\hline Bacci et $\mathrm{al}^{32}$ & $\begin{array}{l}\text { Randomized, } 2 \\
\text { arms }\end{array}$ & 40 & $<40$ & Extremity & Included & 2 & $77 \%$ & NA & $26 \%$ at $5 y$ \\
\hline Bacci et $\mathrm{al}^{32}$ & $\begin{array}{l}\text { Randomized, } 2 \\
\text { arms }\end{array}$ & 72 & $<40$ & Extremity & Included & 2 & $80 \%$ & NA & $61 \%$ at $5 y$ \\
\hline Wilkins et $\mathrm{al}^{37}$ & Single arm & 47 & $<21$ & Extremity & Excluded & $\begin{array}{l}\text { 3-5 (response } \\
\text { dependent) }\end{array}$ & $87 \%$ & $92 \%$ at $10 y$ & $84 \%$ at $10 y$ \\
\hline Wilkins et $\mathrm{al}^{36}$ & Single arm & 62 & $<22$ & Extremity & Excluded & $\begin{array}{l}\text { 3-5 (response } \\
\text { dependent) }\end{array}$ & $87 \%$ & $93 \%$ at $10 y$ & $86 \%$ at $10 y$ \\
\hline Xie et $\mathrm{al}^{13}$ & $\begin{array}{l}\text { Nonrandomized, } \\
2 \text { arms }\end{array}$ & 48 & NA & Extremity & Included & 3 & $63 \%$ & $64 \%$ at $5 y$ & $60 \%$ at $5 y$ \\
\hline $\begin{array}{l}\text { Hu et al } \\
\text { (Current } \\
\text { study) }\end{array}$ & Single arm & 12 & $11 \sim 69$ & Pelvis & Excluded & 2 & $66.7 \%$ & $57.8 \%$ at $5 y$ & $52.5 \%$ at $5 y$ \\
\hline
\end{tabular}

Abbreviations: IA, intraarterial; CDDP, cisplatin; TN, tumor necrosis; OS, overall survival; EFS, event-free survival. 
was insufficient to test the $9 \%$ difference between the two groups. $^{35}$ In the multicenter study reported by Winkler et al and Fuchs et al in the 1990s, no difference in chemotherapy response was observed between the intraarterial and IV CDDP groups. ${ }^{30,34}$ Similarly, Xie et al also proved a superior histological response rate but equivalent survival in the CAI group versus the IV group in $2019 .{ }^{13}$ However, the reliability of the conclusions in the 2 studies may be impaired by the nonrandomized grouping design conducted at the researchers' discretion, which could be affected by the severity of disease. Although CAI may bring benefits to chemotherapy response augmentation and survival rates in limb osteosarcoma patients, how pelvic osteosarcoma reacts to CAI-facilitated multidrug chemotherapy remains elusive.

Pelvic tumor resection surgeries such as hemipelvectomy are notoriously known as one of the most destructive operations in modern orthopedic surgery. ${ }^{38}$ Due to the adjacency of the vascular network, nerves and viscera in the pelvis, surgeons have to weigh tumor eradication against functional preservation in the determination of the extent of surgical excision. This dilemma makes the treatment more challenging. Patients with pelvic osteosarcoma have an approximately 30\% lower survival rate compared with the extremity-morbidity population, which could be explained by the higher rate of not only failure of surgical remission but also advanced age, large tumor size, primary metastasis, prolonged latency periods and poor chemotherapy responses. ${ }^{11,39}$ Thus, it is critical to maximize the chemotherapy response and achieve better survival outcomes in pelvic osteosarcoma patients.

The combination of agents in our MSC protocol was determined by reference to NCCN guidelines for bone cancer and the study reported by Xie et al. ${ }^{13}$ Although the agent numbers and categories differed, all of the above-reviewed studies and the present study deployed a multidrug protocol to treat tumor cells in different phases of the cell cycle. Unfortunately, insufficient data are available to appropriately compare the therapeutic effect of CAI alone or in various combinations with MTX, VCR, $\mathrm{ADM}$ and IFO because of great heterogeneity. In the present study, we used high-dose MTX at $10 \mathrm{~g} / \mathrm{m}^{2}$ and CAI at $120 \mathrm{mg} / \mathrm{m}^{2}$ in 2 doses as routine preoperative treatment. For patients with tumors over $8 \mathrm{~cm}$, we employed an enhanced dose of CAI at $160 \mathrm{mg} / \mathrm{m}^{2}$. Additionally, additional IFO courses were given to slow responders. This individualized regimen directly yielded a $66.7 \%$ prevalence of good histopathologic responders and contributed to the 5-year survival rate of $57.8 \%$ in pelvic osteosarcoma. Both of the two figures were seemingly improved when compared to those from conventional systematic chemotherapy. ${ }^{11}$

The cumulative dosage of CDDP was $780 \mathrm{mg} / \mathrm{m}^{2}$ for a good responder with a tumor size $\leq 8 \mathrm{~cm}$ in the present study. Wilkins et al reported the highest accumulated dose of CDDP at $960 \mathrm{mg} / \mathrm{m}^{2}$ and a single dose at $160 \mathrm{mg} / \mathrm{m}^{2}$ over $24 \mathrm{~h}$ as an enhanced regimen for patients with tumors over $10 \mathrm{~cm}$ versus $120 \mathrm{mg} / \mathrm{m}^{2}$ over $6 \mathrm{~h}$ as routine, which consequently yielded the highest 10-year OS and EFS rates (93\% and 86\%, respectively) among all reviewed studies. Notably, Ferrari et al and Xie et al investigated CDDP administration at equivalent doses to those in the present study but yielded poorer survival outcomes seemingly. ${ }^{13,33}$ This may be because $120 \mathrm{mg} / \mathrm{m}^{2}$ CDDP was given intraarterially in $72 \mathrm{~h}$ in the first study, which is too long to achieve a high enough blood concentration; patients with tumor metastases at the initial diagnosis were included in the second study so that the survival rate would be reduced. We used relatively high-dose CDDP administered over 3 to $6 \mathrm{~h}$ combined with 4 other drugs, including ETOP, and made individualized adjustments to the protocol for patients with high-risk factors. This may have contributed to the $57.8 \%$ OS and $52.5 \%$ EFS rate at 5 years, which is favorable for pelvic osteosarcoma. Moreover, we discovered that the OS and EFS rates in the population with tumors $\leq$ or $>8 \mathrm{~cm}$ showed insignificant differences. Patients who received $\mathrm{R} 0$ resection also shared equivalent OS but higher EFS with R1 patients. These finding suggest that this protocol with riskdependent adjustment in the drug dosage and combination could be beneficial to bridge the gap in survival rate between patients with large or small tumors, and those who received $\mathrm{R} 0$ or $\mathrm{R} 1$ resection.

We noticed that 3 of 5 cases developing SD turned out to demonstrate TNR $>90 \%$, which probably means that good response of osteosarcoma is not necessarily accompanied with tumor size decrease. This may be attributed to the formation of tumor bone generated by subtypes like osteoblastic osteosarcoma, which could prevent tumors from shrinkage. ${ }^{40}$

Only 4 poor responders were observed, 3 of whom developed tumor relapses. Two of the 3 patients died of metastasis during postoperative MSC; another showed no evidence of disease after receiving secondary amputation and resection for local recurrence. This protocol seemed to provide limited benefit to the poor responders. However, 
the present data are so limited that few tenable conclusions can be drawn from it to guide salvage treatment for poor responders.

Although the AI technique and additional courses were given in this protocol, no patients received an overdose of chemotherapy drugs compared with previous studies using similar drug combinations. No AI-relative grade 4 or 5 adverse events were observed. Although systematic adverse events, including nausea, vomiting, anemia and an increase in creatinine/aminotransferase levels, shared equivalent incidence rates and severities with the study reported by Xie et al, local skin hyperpigmentation and pelvic soft tissue necrosis in muscle occurred after only 3 and 1 of 24 CAI events ( $12.5 \%$ and $4.2 \%$, respectively), which is more favorable than the situation reported in the most reviewed studies concerning extremity osteosarcoma. ${ }^{13,30,33}$ This finding could be explained by the shorter local drug-retention time since the pelvis has a more sufficient blood supply and drainage.

In terms of the postoperative MSC regimen for poor responders, we determined the IFO + ETOP regimen by reference to the second-line chemotherapy recommended in NCCN guidelines for bone cancer, which is supported by the study reported by Goorin et al and Miser et al. ${ }^{23,41}$ These two studies were conducted in patients with initial metastasis or recurrence of osteosarcoma, and the former one used IFO + ETOP as an induction regimen accompanied by surgical resection and continuation MSC of MTX + $\mathrm{ADM}+\mathrm{ETOP}+\mathrm{IFO}$. Therefore, we adopted the regimen similar as the one reported by Miser et al However, the study of EURAMOS reported by Marina et al suggested that treatment with MTX + ADM + CDDP + IFO + ETOP resulted in similar EFS to MTX + ADM + CDDP and increased toxicity. In the present study, we found it hard for patients to accept 12 cycles of second-line therapy in 3 years in view of the high expenditure and physical discomfort. Therefore, the number of cycles was reduced to 6 for poor responders, which is equivalent to a half of the regimen of Miser et al in accumulative dosage and duration time. During the postoperative MSC period, there was no early death. The reported cardiac irritation, increase in creatinine/aminotransferase, oral mucosa inflammation and hematologic system abnormality were acceptable. In summary, our chemotherapy regimen seems to be well tolerated.

To monitor the chemotherapy response, several methods have been reported in previous studies. In the studies reported by Winkler et al and Fuch et al, plain X-ray, local $\mathrm{CT}$ and emission $\mathrm{CT}$ of bone were used to monitor the
TNR before the surgery. ${ }^{30,34}$ Bacci et al, Wilkins et al and Xie et al deployed CT arterial angiography, which yielded sensitivity and specificity over $90 \%$ and $50 \%$, respectively. ${ }^{13,32,36,37}$ Nevertheless, a repeated CT arterial angiography assessment is difficult to be accepted by patients because it requires multiple invasive operations and high expenditure. RECIST-1.1-based MRI remains one of the most commonly used approaches in tumor response evaluation. ${ }^{42,43}$ In the present study, we used CEMRI to detect the tumor response and guide the adjustment of treatment for each patient. Although the results did not seem to suggest that this method is highly predictive of the histopathologic chemotherapy response, the equivalent survival rate between patients with large and small tumors may reflect the effectiveness of the individualized treatment.

There were several limitations in our investigation. First, the design was restricted to a small-sample-size single-arm clinical study due to the rarity of pelvis osteosarcoma morbidity, therefore reducing the reliability of the conclusions. Second, several previous studies reported that pathological subtypes of osteosarcoma, such as chondroblastic and telangiectatic osteosarcoma, respond differently to chemotherapy. ${ }^{39,44}$ However, the pathological subtype of each patient was not analyzed as an independent prognostic factor in our study. Heterogeneity of the osteosarcoma subtype constitution could distort chemotherapy response and survival outcomes. Third, we used CE-MRI per RECIST 1.1 to monitor the tumor response before surgery and guide the individualized adjustment of the treatment protocol. However, CE-MRI evaluation was not the gold standard for TNR prediction. Multiple innovative approaches for TNR prediction in osteosarcoma have been researched in the past 30 years, such as dynamic CE-MRI with sensitivity and specificity over $70 \%$ and $80 \%$, respectively, according to previous studies. ${ }^{45-47}$ We would attempt to deploy approaches with higher accuracy to evaluate viable tumors in hope of providing more precise guidance for individualized pelvic osteosarcoma treatment in further studies.

\section{Conclusions}

In the present study, we employed CAI in the multimodal treatment of pelvic osteosarcoma patients. Our findings suggest that the CAI-facilitated chemotherapy regimen demonstrated great potential for improving the survival of patients with pelvic osteosarcoma. This treatment protocol yielded high tolerance with acceptable adverse 
effects. However, further randomized controlled trials with large sample sizes are required to elucidate the findings of this preliminary study.

\section{Abbreviations}

ADM, adriamycin; CAI, effect of cisplatin arterial infusion; CDDP, cis-diamine-dichloro platinum; CE-MRI, contrast-enhanced MRI; CR, complete response; CTCAE, Common Terminology Criteria for Adverse Events; EFS, event-free survival; ETOP, etoposide; IFO, ifosfamide; IV, intravenous; MSC, multidrug systematic chemotherapy; MTX, methotrexate; OS, overall survival; PD, progressive disease; PR, partial response; RECIST, response evaluation criteria in solid tumors version; S, surgery; SD, stable disease; TNR, tumor necrosis rate; VCR, vincristine.

\section{Data Sharing Statement}

The datasets used and/or analyzed during the current study are available from the corresponding author on reasonable request.

\section{Ethics Approval and Informed Consent}

This study was approved by the ethics committees and the institutional review board of the Second Xiangya Hospital. All patients provided written informed consent for receiving this treatment protocol. The requirement of obtaining specific informed consent for this retrospective study was waived for the reason mentioned before.

\section{Consent for Publication}

This study conforms to Declaration of Helsinki. The requirement of obtaining specific informed consent from the patients for the publication of the case presentation and any accompanying images.

\section{Acknowledgments}

We would like to thank all patients for their devotion to taking unidentified risks in this clinical trial. We are also grateful to the participating orthopedic oncologists and interventional radiologists for their prudence and patience in the therapeutic effect evaluation and the multidisciplinary consultation.

\section{Author Contributions}

All authors made a significant contribution to the work reported, whether that is in the conception, study design, execution, acquisition of data, analysis and interpretation, or in all these areas; took part in drafting, revising or critically reviewing the article; gave final approval of the version to be published; have agreed on the journal to which the article has been submitted; and agree to be accountable for all aspects of the work.

\section{Funding}

This study was supported by the Natural Science Foundation of Hunan Province for Youths (Grant number 2020JJ5799).

\section{Disclosure}

No commercial sponsorship was involved in any part of this study. The authors report no conflicts of interest related to this work.

\section{References}

1. Tu C, He J, Qi L, et al. Emerging landscape of circular RNAs as biomarkers and pivotal regulators in osteosarcoma. J Cell Physiol. 2020;235(12):9037-9058. doi:10.1002/jcp.29754

2. Gao SS, Wang YJ, Zhang GX, Zhang WT. Potential diagnostic value of miRNAs in peripheral blood for osteosarcoma: a meta-analysis. J Bone Oncol. 2020;23:100307. doi:10.1016/j.jbo.2020.100307

3. Mirabello L, Troisi RJ, Savage SA. Osteosarcoma incidence and survival rates from 1973 to 2004: data from the surveillance, epidemiology, and end results program. Cancer. 2009;115(7):1531-1543. doi:10.1002/cncr.24121

4. Lagmay JP, Krailo MD, Dang H, et al. Outcome of patients with recurrent osteosarcoma enrolled in seven Phase II trials through children's cancer group, pediatric oncology group, and children's oncology group: learning from the past to move forward. $J$ Clin Oncol. 2016;34(25):3031-3038. doi:10.1200/jco.2015.65.5381

5. Pappo AS, Vassal G, Crowley JJ, et al. A Phase 2 trial of R1507, a monoclonal antibody to the insulin-like growth factor-1 receptor (IGF-1R), in patients with recurrent or refractory rhabdomyosarcoma, osteosarcoma, synovial sarcoma, and other soft tissue sarcomas: results of a sarcoma alliance for research through collaboration study. Cancer. 2014;120(16):2448-2456. doi:10.1002/cncr.28728

6. Xu J, Xie L, Guo W. Neoadjuvant chemotherapy followed by delayed surgery: is it necessary for all patients with nonmetastatic high-grade pelvic osteosarcoma? Clin Orthop Relat Res. 2018;476 (11):2177-2186. doi:10.1097/corr.0000000000000387

7. Eilber FR, Mirra JJ, Grant TT, Weisenburger T, Morton DL. Is amputation necessary for sarcomas? A seven-year experience with limb salvage. Ann Surg. 1980;192(4):431-438. doi:10.1097/000006 58-198010000-00001

8. Link MP, Goorin AM, Horowitz M, et al. Adjuvant chemotherapy of high-grade osteosarcoma of the extremity. Updated results of the multi-institutional osteosarcoma study. Clin Orthop Relat Res. $1991 ;(270): 8-14$.

9. Bacci G, Longhi A, Fagioli F, Briccoli A, Versari M, Picci P. Adjuvant and neoadjuvant chemotherapy for osteosarcoma of the extremities: 27 year experience at Rizzoli Institute, Italy. Eur $J$ Cancer. 2005;41(18):2836-2845. doi:10.1016/j.ejca.2005.08.026

10. Ferrari S, Meazza C, Palmerini E, et al. Nonmetastatic osteosarcoma of the extremity. neoadjuvant chemotherapy with methotrexate, cisplatin, doxorubicin and ifosfamide. An Italian Sarcoma group study (ISG/OS-Oss). Tumori J. 2014;100(6):612-619. doi:10.1177/1778. 19262 
11. Bielack SS, Kempf-Bielack B, Delling G, et al. Prognostic factors in high-grade osteosarcoma of the extremities or trunk: an analysis of 1702 patients treated on neoadjuvant cooperative osteosarcoma study group protocols. J Clin Oncol. 2002;20(3):776-790. doi:10.1200/ jco.2002.20.3.776

12. Rosenberg B. Platinum coordination complexes in cancer chemotherapy. Naturwissenschaften. 1973;60(9):399-406. doi:10.1007/bf00623551

13. Xie L, Xu J, Dong S, et al. Gain and loss from transcatheter intra-arterial limb infusion of cisplatin for extremity osteosarcoma: a retrospective study of 99 cases in the past six years. Cancer Manag Res. 2019;11:7183-7195. doi:10.2147/cmar.S214604

14. Cullen JW, Jamroz BA, Stevens SL, et al. The value of serial arteriography in osteosarcoma: delivery of chemotherapy, determination of therapy duration, and prediction of necrosis. $J$ Vasc Interv Radiol. 2005;16(8):1107-1119. doi:10.1097/01.Rvi.0000167856.31 329.F8

15. Abe S, Nishimoto Y, Isu K, Ishii T, Goto T. Preoperative cisplatin for initial treatment of limb osteosarcoma: its local effect and impact on prognosis. Cancer Chemother Pharmacol. 2002;50(4):320-324. doi:10.1007/s00280-002-0501-Z

16. Fox E, Levin K, Zhu Y, et al. Pantoprazole, an inhibitor of the organic cation transporter 2 , does not ameliorate cisplatin-related ototoxicity or nephrotoxicity in children and adolescents with newly diagnosed osteosarcoma treated with methotrexate, doxorubicin, and cisplatin. Oncologist. 2018;23(7):762-e79. doi:10.1634/theoncologist.2018-0037

17. van der Woude HJ, Bloem JL, Verstraete KL, Taminiau AH, Nooy MA, Hogendoorn PC. Osteosarcoma and Ewing's sarcoma after neoadjuvant chemotherapy: value of dynamic MR imaging in detecting viable tumor before surgery. AJR Am J Roentgenol. 1995;165(3):593-598. doi:10.2214/ajr.165.3.7645476

18. Lai YC, Chang WC, Chen CB, et al. Response evaluation for immunotherapy through semi-automatic software based on RECIST 1.1, irRC, and iRECIST criteria: comparison with subjective assessment. Acta Radiol. 2020;61(7):983-991. doi:10.1177/ 0284185119887588

19. Kitajima K, Miyoshi Y, Yamano T, Odawara S, Higuchi T, Yamakado K. Assessment of tumor response to neoadjuvant chemotherapy in patients with breast cancer using MRI and FDG-PET/ CT-RECIST 1.1 vs. PERCIST 1.0. Nagoya J Med Sci. 2018;80 (2):183-197. doi:10.18999/nagjms.80.2.183

20. Marina NM, Smeland S, Bielack SS, et al. Comparison of MAPIE versus MAP in patients with a poor response to preoperative chemotherapy for newly diagnosed high-grade osteosarcoma (EURAMOS-1): an open-label, international, randomised controlled trial. Lancet Oncol. 2016;17(10):1396-1408. doi:10.1016/s1470-20 45(16)30214-5

21. Wang H, Tang X, Xie L, Dong S, Chen C, Guo W. Stop-flow pelvic chemoperfusion for the treatment of malignant pelvic bone tumors: a preliminary study. Orthop Surg. 2020;12(3):741-748. doi:10.1111/ os. 12666

22. Huvos AG, Rosen G, Marcove RC. Primary osteogenic sarcoma: pathologic aspects in 20 patients after treatment with chemotherapy en bloc resection, and prosthetic bone replacement. Arch Pathol Lab Med. 1977;101(1):14-18.

23. Miser JS, Kinsella TJ, Triche TJ, et al. Ifosfamide with mesna uroprotection and etoposide: an effective regimen in the treatment of recurrent sarcomas and other tumors of children and young adults. J Clin Oncol. 1987;5(8):1191-1198. doi:10.1200/jco.1987.5.8.1191

24. Callaghan CM, Seyedin SN, Mohiuddin IH, et al. The effect of concurrent stereotactic body radiation and anti-PD-1 therapy for recurrent metastatic sarcoma. Radiat Res. 2020;194(2):124-132. doi:10.1667/rade-20-00017

25. Evans AE. The success and failure of multimodal therapy for cancer in children. Cancer. 1975;35(1):48-54. doi:10.1002/1097-0142(19 7501)35:1<48::aid-cncr2820350107>3.0.co;2-b
26. Harrison DJ, Geller DS, Gill JD, Lewis VO, Gorlick R. Current and future therapeutic approaches for osteosarcoma. Expert Rev Anticancer Ther. 2018;18(1):39-50. doi:10.1080/14737140.2018.1413939

27. Smeland S, Bielack SS, Whelan J, et al. Survival and prognosis with osteosarcoma: outcomes in more than 2000 patients in the EURAMOS-1 (European and American Osteosarcoma Study) cohort. Eur J Cancer. 2019;109:36-50. doi:10.1016/j.ejca.2018.11.027

28. Zhang Y, Yang J, Zhao N, et al. Progress in the chemotherapeutic treatment of osteosarcoma. Oncol Lett. 2018;16(5):6228-6237. doi:10.3892/ol.2018.9434

29. Stephens FO. Induction chemotherapy: to downgrade aggressive cancers to improve curability by surgery and/or radiotherapy. Eur J Surg Oncol. 2001;27(7):672-688. doi:10.1053/ejso.2001.1158

30. Winkler K, Bielack S, Delling G, et al. Effect of intraarterial versus intravenous cisplatin in addition to systemic doxorubicin, high-dose methotrexate, and ifosfamide on histologic tumor response in osteosarcoma (study COSS-86). Cancer. 1990;66(8):1703-1710. doi:10.1002/ 1097-0142(19901015)66:8<1703::aid-cncr2820660809>3.0.co;2-v

31. Jaffe N, Knapp J, Chuang VP, et al. Osteosarcoma: intra-arterial treatment of the primary tumor with cis-diammine-dichloroplatinum II (CDP). Angiographic, pathologic, and pharmacologic studies. Cancer. 1983;51(3):402-407. doi:10.1002/1097-0142(19830201) 51:3<402::aid-cncr2820510308>3.0.co;2-p

32. Bacci G, Ferrari S, Tienghi A, et al. A comparison of methods of loco-regional chemotherapy combined with systemic chemotherapy as neo-adjuvant treatment of osteosarcoma of the extremity. Eur J Surg Oncol. 2001;27(1):98-104. doi:10.1053/ejso. 2000.1056

33. Ferrari S, Mercuri M, Picci P, et al. Nonmetastatic osteosarcoma of the extremity: results of a neoadjuvant chemotherapy protocol (IOR/ OS-3) with high-dose methotrexate, intraarterial or intravenous cisplatin, doxorubicin, and salvage chemotherapy based on histologic tumor response. Tumori. 1999;85(6):458-464. doi:10.1177/030089 169908500607

34. Fuchs N, Bielack SS, Epler D, et al. Long-term results of the cooperative German-Austrian-Swiss osteosarcoma study group's protocol COSS-86 of intensive multidrug chemotherapy and surgery for osteosarcoma of the limbs. Ann Oncol. 1998;9(8):893-899. doi:10. 1023/a:1008391103132

35. Rha SY, Chung HC, Gong SJ, et al. Combined pre-operative chemotherapy with intra-arterial cisplatin and continuous intravenous adriamycin for high grade osteosarcoma. Oncol Rep. 1999;6 (3):631-637. doi:10.3892/or.6.3.631

36. Wilkins RM, Cullen JW, Camozzi AB, Jamroz BA, Odom L. Improved survival in primary nonmetastatic pediatric osteosarcoma of the extremity. Clin Orthop Relat Res. 2005;\&NA;(438):128-136. doi:10.1097/01.blo.0000179736.10871.76

37. Wilkins RM, Cullen JW, Odom L, et al. Superior survival in treatment of primary nonmetastatic pediatric osteosarcoma of the extremity. Ann Surg Oncol. 2003;10(5):498-507. doi:10.1245/aso. 2003.03.061

38. van Houdt WJ, Griffin AM, Wunder JS, Ferguson PC. Oncologic outcome and quality of life after hindquarter amputation for sarcoma: is it worth it? Ann Surg Oncol. 2018;25(2):378-386. doi:10.1245/ s10434-017-5806-6

39. Song WS, Cho WH, Jeon DG, et al. Pelvis and extremity osteosarcoma with similar tumor volume have an equivalent survival. $J$ Surg Oncol. 2010;101(7):611-617. doi:10.1002/jso.21540

40. Liu $\mathrm{C}, \mathrm{Xi} \mathrm{Y,} \mathrm{Li} \mathrm{M,} \mathrm{et} \mathrm{al.} \mathrm{Monitoring} \mathrm{response} \mathrm{to} \mathrm{neoadjuvant}$ chemotherapy of primary osteosarcoma using diffusion kurtosis magnetic resonance imaging: initial findings. Korean J Radiol. 2019;20 (5):801-811. doi:10.3348/kjr.2018.0453

41. Goorin AM, Harris MB, Bernstein M, et al. Phase II/III trial of etoposide and high-dose ifosfamide in newly diagnosed metastatic osteosarcoma: a pediatric oncology group trial. J Clin Oncol. 2002;20 (2):426-433. doi:10.1200/jco.2002.20.2.426 
42. Eisenhauer EA, Therasse P, Bogaerts J, et al. New response evaluation criteria in solid tumours: revised RECIST guideline (version 1.1). Eur $J$ Cancer. 2009;45(2):228-247. doi:10.1016/j.ejca.2008.10.026

43. Tawbi HA, Burgess M, Bolejack V, et al. Pembrolizumab in advanced soft-tissue sarcoma and bone sarcoma (SARC028): a multicentre, two-cohort, single-arm, open-label, phase 2 trial. Lancet Oncol. 2017;18(11):1493-1501. doi:10.1016/s1470-2045(17)30624-1

44. Fahey M, Spanier SS, Vander Griend RA. Osteosarcoma of the pelvis. A clinical and histopathological study of twenty-five patients. J Bone Joint Surg Am. 1992;74(3):321-330. doi:10.2106/ 00004623-199274030-00002

45. Erlemann R, Sciuk J, Bosse A, et al. Response of osteosarcoma and Ewing sarcoma to preoperative chemotherapy: assessment with dynamic and static MR imaging and skeletal scintigraphy. Radiology. 1990;175(3):791-796. doi:10.1148/radiology.175.3.2188300
46. Torricelli P, Montanari N, Spina V, et al. Dynamic contrast enhanced magnetic resonance imaging subtraction in evaluating osteosarcoma response to chemotherapy. Radiol Med. 2001;101(3):145-151.

47. Kubo T, Furuta T, Johan MP, Adachi N, Ochi M. Percent slope analysis of dynamic magnetic resonance imaging for assessment of chemotherapy response of osteosarcoma or Ewing sarcoma: systematic review and meta-analysis. Skeletal Radiol. 2016;45(9):1235-1242. doi:10.1007/s00256-016-2410-y

\section{Publish your work in this journal}

Cancer Management and Research is an international, peer-reviewed open access journal focusing on cancer research and the optimal use of preventative and integrated treatment interventions to achieve improved outcomes, enhanced survival and quality of life for the cancer patient.
The manuscript management system is completely online and includes a very quick and fair peer-review system, which is all easy to use. Visit http://www.dovepress.com/testimonials.php to read real quotes from published authors. 